\title{
Exotic Solutions in Einstein-Antisymmetric Tensor Theory
}

\author{
Hongsu Kim \\ Department of Physics \\ Sogang University, C.P.O. Box 1142, Seoul 100-611, KOREA
}

(August, 1995)

\begin{abstract}
Classical solutions of the self-interacting, non-abelian antisymmetric tensor gauge theory of Freedman and Townsend coupled to Einstein gravity is discussed. Particularly, it is demonstrated that the theory admits a classical metric solution which, depending on the value of the gauge coupling parameter of the theory, exhibits a black hole with an exotic non-abelian hair or a spacetime showing the "violation of the cosmic censorship hypothesis" which should be distinguished from white holes.
\end{abstract}

PACS numbers: 11.15.-q, 04.20.Jb, 04.50.+h, 04.65.+e

Keywords: Antisymmetric tensor, Einstein gravity, Black Hole, Cosmic Censorship 
In this letter, we consider the classical solutions of the antisymmetric tensor gauge theory coupled to the Einstein gravity. Generally, the antisymmetric tensor field occurs in some formulations of supergravity theories, relativistic strings, etc[1-3]. In particular, a model of a self-interacting antisymmetric tensor field possessing a non-abelian gauge invariance was first discussed by Freedman and Townsend (FT)[4] in which they established, among other things, the on-shell equivalence between the antisymmetric tensor gauge theory and the nonlinear sigma model. Subsequent works attempting the quantizaion of the self-interacting antisymmetric tensor theory of FT also have appeared in the literature [5]. As for the motivation for the present work, we are interested in a rather different aspect of the theory; we look for the classical metric and matter solutions of the "Einstein-Antisymmetric tensor (EAT)" theory. As the main result, we will demonstrate that the theory admits a classical metric solution which, depending on the value of the gauge coupling parameter of the theory, exhibits a black hole with a non-abelian hair or a spacetime showing the "violation of the cosmic censorship hypothesis." Before formualting our theory, we begin by briefly reviewing the antisymmetric tensor gauge theory in flat Minkowski spacetime [4]. The self-interacting, non-abelian antisymmetric tensor gauge theory of FT can be formulated in its second-order version or alternatively in its first-order version $[4,5]$. And the two formulations are, of course, on-shell equivalent. For some purposes, the "first-order formulation" of the theory is convenient. In this formulation, the action is given by

$$
L=\frac{1}{8} \epsilon^{\mu \nu \rho \sigma} B_{\mu \nu}^{a} F_{\rho \sigma}^{a}-\frac{1}{8} A_{\mu}^{a} A^{a \mu}
$$

where the antisymmetric tensor gauge field $B_{\mu \nu}^{a}$ and a vector potential $A_{\mu}^{a}$ associated with the field strength $F_{\mu \nu}^{a}$ are treated as being independent variables of the theory. Now by varying this Lagrangian with respect to these independent variables, we can obtain the classical field equation for $B_{\mu \nu}^{a}$ and $A_{\mu}^{a}$ respectively as

$$
\begin{aligned}
& F_{\mu \nu}^{a}=0, \\
& D^{\mu} \tilde{B}_{\mu \nu}^{a}+A_{\nu}^{a}=0
\end{aligned}
$$




$$
\begin{aligned}
& \text { where } \begin{aligned}
F_{\mu \nu}^{a} & =\partial_{\mu} A_{\nu}^{a}-\partial_{\nu} A_{\mu}^{a}+g_{c} f^{a b c} A_{\mu}^{b} A_{\nu}^{c} \\
D_{\mu}^{a c} & =\left(\partial_{\mu} \delta^{a c}+g_{c} f^{a b c} A_{\mu}^{b}\right), \text { and } \tilde{\mathrm{B}}_{\mu \nu}^{\mathrm{a}}=\epsilon_{\mu \nu}^{\rho \sigma} \mathrm{B}_{\rho \sigma}^{\mathrm{a}} .
\end{aligned}
\end{aligned}
$$

Further, by acting the operator $D^{\nu}$ on the field equation for $A_{\mu}^{a}$ in eq.(3) and using eq.(2), we get

$$
D^{\mu} A_{\mu}^{a}=0
$$

Then the vector potential $A_{\mu}^{a}$ satisfying classical field equation eqs.(2) and (3) turns out to be the 'pure gauge',

$$
A_{\mu}=-\frac{i}{g_{c}}\left(\partial_{\mu} U(x)\right) U^{-1}(x)
$$

(where $U(x)$ is the gauge tranformation function of the given non-abelian gauge group) provided it (eq.(5)) satisfies the "combined" field equation (4). Using the classical field equation for $A_{\mu}^{a}$ in eq.(3) one can show that the first-order formulation Lagrangian in eq.(1) turns into the second-order formulation Lagrangian [4]. Further, since the classical field equation for $B_{\mu \nu}^{a}$ is the vanishing $F_{\mu \nu}^{a}$, in this first-order formulation $B_{\mu \nu}^{a}$ field appears classically as a Lagrange multiplier enforcing the constraint $F_{\mu \nu}^{a}=0$. And finally, substituting the pure gauge solution in eq.(5) into the Lagrangian (1) demonstrates the equivalence of the theory to the non-linear $\sigma$-model [4]. Now we consider the case when the gravity is turned on. To begin, it seems essential for us to declare our sign convention. We choose to take the convention in which $g_{\mu \nu}=\operatorname{diag}(-+++)$ and $R_{\beta \mu \nu}^{\alpha}=\partial_{\mu} \Gamma_{\beta \nu}^{\alpha}-\partial_{\nu} \Gamma_{\beta \mu}^{\alpha}+\Gamma_{\mu \lambda}^{\alpha} \Gamma_{\beta \nu}^{\lambda}-\Gamma_{\nu \lambda}^{\alpha} \Gamma_{\beta \mu}^{\lambda}$. It is crucial to fix the right sign for the antisymmetric tensor (i.e., the matter) sector of the action "relative" to the gravity action. Therefore in our sign convention, we explain the way we determined the sign for the matter action using the fact that on-shell, the antisymmetric tensor theory action is equivalent to that of non-linear sigma model with the right sign. Consider generators $T^{a}$ of the non-abelian group $G$ in a representation in which $\left[T^{a}, T^{b}\right]=i f^{a b c} T^{c}, \operatorname{Tr}\left(T^{a} T^{b}\right)=c \delta^{a b}$ and $U(x)=\exp \left[i \phi^{a}(x) T^{a}\right]$ where $f^{a b c}$ and $c$ are the structure constant and a representation-dependent positive constant respectively. Then it can be readily shown that upon substituting the on-shell condition, $F_{\mu \nu}=0$, i.e., 
$A_{\mu}=-\frac{i}{g_{c}}\left(\partial_{\mu} U\right) U^{-1}$, one gets $L=\left(\frac{1}{8 c}\right) \operatorname{Tr}\left[\epsilon^{\mu \nu \rho \sigma} B_{\mu \nu} F_{\rho \sigma}-A_{\mu} A^{\mu}\right]=\left(-\frac{1}{8 c g_{c}^{2}}\right) \operatorname{Tr}\left[\left(\partial_{\mu} U^{-1}\right)\left(\partial^{\mu} U\right)\right]$ (with $B_{\mu \nu}=B_{\mu \nu}^{a} T^{a}$ and $A_{\mu}=A_{\mu}^{a} T^{a}$ ) which is of the right sign. Thus in this way we have fixed the sign for the matter action. Now in order to describe the coupled Einstein-Antisymmetric tensor theory we again employ the first order formulation of the antisymmetric tensor sector, then the theory is described by the action (we work in the unit $G=1$ )

$$
\begin{aligned}
S & =S_{G}+S_{A T} \\
& =\int d^{4} x \sqrt{g}\left[\frac{1}{16 \pi} R+\frac{1}{8}\left(\frac{1}{\sqrt{g}} g^{\mu \alpha} g^{\nu \beta} \tilde{B}_{\mu \nu}^{a} F_{\alpha \beta}^{a}-g^{\mu \nu} A_{\mu}^{a} A_{\nu}^{a}\right)\right]
\end{aligned}
$$

where we used that in curved spacetime, $\epsilon^{\mu \nu \rho \sigma} \rightarrow\left(\frac{\epsilon^{\mu \nu \rho \sigma}}{\sqrt{g}}\right)$ and again $\tilde{B}_{\mu \nu}^{a}=\epsilon_{\mu \nu}{ }^{\rho \sigma} B_{\rho \sigma}^{a}=$ $g_{\mu \alpha} g_{\nu \beta} \epsilon^{\alpha \beta \rho \sigma} B_{\rho \sigma}^{a}$. The curved spacetime version of the classical field equation for $B_{\mu \nu}^{a}$ and $A_{\mu}^{a}$ are given respectively by

$$
\begin{aligned}
& F_{\mu \nu}^{a}=0, \\
& D^{\mu} \tilde{B}_{\mu \nu}^{a}+\sqrt{g} A_{\nu}^{a}=0
\end{aligned}
$$

along with the curved spacetime counterpart of the eq.(4) which is the necessary condition that $A_{\mu}^{a}$ must satisfy as a classical solution being given by

$$
D^{\mu}\left(\sqrt{g} A_{\mu}^{a}\right)=0 .
$$

In addition, varying the action in eq.(6) with respect to the metric $g_{\mu \nu}$ yields the Einstein field equation

$$
R_{\mu \nu}=-4 \pi\left[\frac{1}{\sqrt{g}}\left\{g^{\alpha \beta}\left(\tilde{B}_{\mu \alpha}^{a} F_{\nu \beta}^{a}\right)-\frac{1}{2} g_{\mu \nu}\left(\tilde{B}_{\alpha \beta}^{a} F^{a \alpha \beta}\right)\right\}-\frac{1}{2}\left(A_{\mu}^{a} A_{\nu}^{a}\right)\right]
$$

with the energy-momemtum tensor being given by

$$
T_{\mu \nu}=-\frac{1}{8}\left[\frac{1}{\sqrt{g}} g^{\alpha \beta}\left(4 \tilde{B}_{\mu \alpha}^{a} F_{\nu \beta}^{a}\right)+\left\{g_{\mu \nu}\left(A_{\alpha}^{a} A^{a \alpha}\right)-2\left(A_{\mu}^{a} A_{\nu}^{a}\right)\right\}\right] .
$$

Our strategy for solving the classical equation of motion in eqs.(7),(8) and (10) along with the necessary condition eq.(9) is as follows ; we start with the solution to the field equations (7) and (9) which, as we shall see, still turns out to be the pure gauge in eq.(5) even in 
the curved spacetime. Next, for this pure gauge solution satisfying eqs.(7) and (9), the Einstein field equation in (10) takes a remarkably simple form, $R_{\mu \nu}=2 \pi\left(A_{\mu}^{a} A_{\nu}^{a}\right)$ with $T_{\mu \nu}=$ $\frac{1}{4}\left[A_{\mu}^{a} A_{\nu}^{a}-\frac{1}{2} g_{\mu \nu}\left(A_{\alpha}^{a} A^{a \alpha}\right)\right]$ that can be readily solvable. Finally by substituting the pure gauge solution for $A_{\nu}^{a}$ and the metric solution $g_{\mu \nu}$ into the field equation (8) (plus possibly the gauge condition of the form $D_{\mu} B^{\mu \nu}=0$ ), one can, in principle, obtain the classical solution for $B_{\mu \nu}^{a}$. We will not, however, expilicitly solve for $B_{\mu \nu}^{a}$ here, partly because we are essentially interested in the spacetime metric solution which, as mentioned, is independent of the solution form of $B_{\mu \nu}^{a}$ and partly because $B_{\mu \nu}^{a}$ appears classically as a Lagrange multiplier enforcing the constraint $F_{\mu \nu}^{a}=0$. Now suppose we look for static, spherically-symmetric solutions to the classical field equations that are also asymptotically flat. Then, first the metric can be written in the form

$$
d s^{2}=-B(r) d t^{2}+A(r) d r^{2}+r^{2} d \Omega_{2}^{2}
$$

with $d \Omega_{2}^{2}$ being the metric on the unit two-sphere. Next, for the matter sector, especially for the vector potential solution of the pure gauge form in eq. (5), in order to look for a spherically-symmetric solution we take the standard ansatz which is the same in form as the flat spacetime Wu-Yang monopole solution ansatz [6] (with the non-abelian gauge group for the antisymmetric tensor field being chosen to be $\mathrm{SU}(2))$

$$
\begin{aligned}
& A_{0}^{a}(r)=0, \\
& A_{i}^{a}(r)=-\epsilon_{i a b} \frac{x^{b}}{g_{c} r^{2}}[1-u(r)] .
\end{aligned}
$$

As is well-known, this solution ansatz is indeed spherically-symmetric in the sense that the effect of a spatial rotation, $\mathrm{SO}(3)$ can be compensated by a gauge transformation, $\mathrm{SU}(2)$. In the spherical-polar coordinates, this ansatz for the vector potential $A_{\mu}^{a}$ and the non-vanishing components of the corresponding field strength $F_{\mu \nu}^{a}$ are given by

$$
A_{0}^{a}=A_{r}^{a}=0, \quad A_{\theta}^{a}=-\frac{1}{g_{c}}[1-u(r)] \hat{\phi}^{a}, \quad A_{\phi}^{a}=\frac{1}{g_{c}}[1-u(r)] \sin \theta \hat{\theta}^{a}
$$

and 


$$
F_{r \theta}^{a}=\frac{u^{\prime}(r)}{g_{c}} \hat{\phi}^{a}, \quad F_{r \phi}^{a}=-\frac{u^{\prime}(r)}{g_{c}} \sin \theta \hat{\theta}^{a}, \quad F_{\theta \phi}^{a}=\frac{\left[u^{2}(r)-1\right]}{g_{c}^{2}} \sin \theta \hat{r}^{a}
$$

where prime denotes the derivative with respect to $r$ and

$$
\begin{aligned}
\hat{r}^{a} & =(\sin \theta \cos \phi, \sin \theta \sin \phi, \cos \theta), \\
\hat{\theta}^{a} & =(\cos \theta \cos \phi, \cos \theta \sin \phi,-\sin \theta), \\
\hat{\phi}^{a} & =(-\sin \phi, \cos \phi, 0) .
\end{aligned}
$$

Here, it is interesting to note that, the case $u(r)=0$ corresponds to the exact albeit singular monopole solution of $\mathrm{Wu}$-Yang type with nonvanishing $A_{\mu}^{a}$ and $F_{\mu \nu}^{a}$; the case $u(r)=+1$ corresponds to the 'trivial' vacuum solution with vanishing $A_{\mu}^{a}$ and $F_{\mu \nu}^{a}$; and finally the case $u(r)=-1$ corresponds to a 'non-trivial' vacuum solution with vanishing $F_{\mu \nu}^{a}$ but nonvanishing $A_{\mu}^{a}$. Therefore, since we are looking for a non-trivial pure gauge solution satisfying $F_{\mu \nu}^{a}=0$, we should take the last case with $u(r)=-1$. Further one can easily check that this non-trivial vacuum gauge solution $A_{0}^{a}=0, A_{i}^{a}=-\epsilon_{i a b}\left(2 x^{b} / g_{c} r^{2}\right)$ does satisfy the necessary condition that it must satisfy, $D^{\mu}\left(\sqrt{g} A_{\mu}^{a}\right)=0$ in eq.(9). Now that we have established the spherically-symmetric vector potential solution to field equations in curved spacetime. As mentioned earlier, then, our next job is to substitute this non-trivial vector potential solution into the Einstein field equation in (10) to solve for the spacetime metric solution. The resulting Einstein equation now reads,

$$
\begin{aligned}
R_{\mu \nu} & =2 \pi\left(A_{\mu}^{a} A_{\nu}^{a}\right), \\
T_{\mu \nu} & =\frac{1}{4}\left[A_{\mu}^{a} A_{\nu}^{a}-\frac{1}{2} g_{\mu \nu}\left(A_{\alpha}^{a} A^{a \alpha}\right)\right], \\
\text { with } \quad \mathrm{A}_{0}^{\mathrm{a}} & =A_{r}^{a}=0, \quad A_{\theta}^{a}=-\frac{2}{g_{c}} \hat{\phi}^{a}, \quad A_{\phi}^{a}=\frac{2}{g_{c}} \sin \theta \hat{\theta}^{a} .
\end{aligned}
$$

Note that in terms of the isotropic metric given in eq.(11) only two components of the Einstein equations out of the three are truely independent because the third component is satisfied automatically due to the energy-momemtum conservation, $T_{; \mu}^{\mu \nu}=0$. Thus we consider the following two independent combinations convenient for solving the Einstein equations, 


$$
\begin{gathered}
\frac{1}{A B}\left(A R_{t t}+B R_{r r}\right)=8 \pi\left[-T_{t}^{t}+T_{r}^{r}\right], \\
\frac{1}{2}\left(\frac{1}{B} R_{t t}+\frac{1}{A} R_{r r}\right)+\frac{1}{r^{2}} R_{\theta \theta}=8 \pi\left[-T_{t}^{t}\right] .
\end{gathered}
$$

The first combination yields $B(r)=A^{-1}(r)$ where we imposed the asymptotic flatness condition, $A(r) \rightarrow 1, B(r) \rightarrow 1$ as $r \rightarrow \infty$. On the other hand the second combination gives

$$
A(r)=\left[1-\frac{2 M(r)}{r}\right]^{-1}
$$

where $M(r)$ is to be determined from $\frac{d M(r)}{d r}=4 \pi r^{2} \rho_{m}(r)=\left(4 \pi / g_{c}^{2}\right)$ with $\rho_{m}(r)=\left[-T_{t}^{t}\right]$. Namely, $M(r)=M+4 \pi r / g_{c}^{2}$ with the integration constant $M$ being identified with the total mass-energy of the system defined at the spatial infinity, $i^{0}$, namely the "ADM mass". Finally, the classical vector potential and the metric solution are given by

$$
\begin{aligned}
A & =A_{\mu} d x^{\mu}=\frac{1}{g_{c}}\left[-2 \tau_{\phi} d \theta+2 \sin \theta \tau_{\theta} d \phi\right], \\
d s^{2} & =-\left[\left(1-\frac{8 \pi}{g_{c}^{2}}\right)-\frac{2 M}{r}\right] d t^{2}+\left[\left(1-\frac{8 \pi}{g_{c}^{2}}\right)-\frac{2 M}{r}\right]^{-1} d r^{2}+r^{2} d \Omega_{2}^{2}
\end{aligned}
$$

where $\tau_{r} \equiv \hat{r}^{a}\left(\sigma^{a} / 2\right), \tau_{\theta}=\hat{\theta}^{a}\left(\sigma^{a} / 2\right), \tau_{\phi}=\hat{\phi}^{a}\left(\sigma^{2} / 2\right)$ with $\sigma^{a}$ being the Pauli spin matrices. Note that there is also a trivial vacuum solution with corresponding gauge potential and metric being given by $A_{\mu}=0($ or $u(r)=+1)$ and the usual Schwarzschild solution respectively. Here it is interesting to recognize that although the two gauge potential solutions, trivial vacuum $A_{\mu}=0$ and the nontrivial vacuum gauge $A_{\mu}=\left(-i / g_{c}\right)\left(\partial_{\mu} U\right) U^{-1}$, are related by a gauge transformation and hence produce the same field strength tensor $F_{\mu \nu}=0$, the spacetime metrics generated by each of the two gauge choices above are not related by any coordinate transformation and thus produce distinct curvatures. This can be easily seen by evaluating the curvature invariant $I=R_{a b c d} R^{a b c d}$ with $a, b, c, d$ being indices associated with an orthonormal basis. In the same Schwarzschild coordinates, the curvature invariant of the usual Schwarzschild solution is given by $I=\frac{48 M^{2}}{r^{6}}$ whereas that of the metric solution in eq.(15) turns out to be $I=16\left[2+\left(1+\frac{4 \pi r}{g_{c}^{2} M}\right)^{2}\right] \frac{M^{2}}{r^{6}}$. Now, we would like to examine the nature of the spacetime described by our metric solution in eq.(15). To do so we consider three cases :

(i) In the weak coupling limit $\left(g_{c}<<2 \sqrt{2 \pi}\right)$; 


$$
d s^{2}=\left[\left(\frac{8 \pi}{g_{c}^{2}}-1\right)+\frac{2 M}{r}\right] d t^{2}-\left[\left(\frac{8 \pi}{g_{c}^{2}}-1\right)+\frac{2 M}{r}\right]^{-1} d r^{2}+r^{2} d \Omega_{2}^{2}
$$

This metric represents a spacetime in which $r$ is timelike and $t$ is spacelike. Thus the metric has an explicit time-dependence. The curvature singularity at $r=0$ is timelike and the future of any Cauchy surface contains a naked singularity which is visible from the future null infinity $I^{+}$. Namely no event horizon arises and thus it exhibits an example of the violation of cosmic censorship hypothesis. Another peculiar characteristic of this spacetime is that when one examine its timelike geodesics, one finds that they involve attractive centrifugal potentials rather than repulsive barriers.

(ii) For the coupling constant $g_{c}=2 \sqrt{2 \pi}$;

$$
d s^{2}=\frac{2 M}{r} d t^{2}-\frac{r}{2 M} d r^{2}+r^{2} d \Omega_{2}^{2}
$$

Again this metric represents a spacetime in which $r$ is timelike and $t$ is spacelike. Also $r=0$ is a naked singularity with no event horizon whatsoever around it and hence leads to the violation of the cosmic censorship hypothesis.

(iii) In the strong coupling limit $\left(g_{c}>>2 \sqrt{2 \pi}\right)$;

$$
d s^{2}=-\left[\left(1-\frac{8 \pi}{g_{c}^{2}}\right)-\frac{2 M}{r}\right] d t^{2}+\left[\left(1-\frac{8 \pi}{g_{c}^{2}}\right)-\frac{2 M}{r}\right]^{-1} d r^{2}+r^{2} d \Omega_{2}^{2} .
$$

This metric describes a black hole spacetime with an event horizon placed at $r=2 M(1-$ $\left.\frac{8 \pi}{g_{c}^{2}}\right)^{-1}$ which encloses a spacelike curvature singularity at $r=0$. Since this metric is characterized by two parameters, $M$ and the non-abelian gauge coupling constant $g_{c}$, the black hole has a non-abelian hair. This black hole spacetime is, as emphasized, not merely a coordinate transformation of the usual Schwarzschild black hole but they have analogous global structures and thermodynamic properties. For instance, this black hole has Hawking temperature and entropy of $T_{H}=\left(1-\frac{8 \pi}{g_{c}^{2}}\right)^{2} / 8 \pi M$ and $S=4 \pi M^{2}\left(1-\frac{8 \pi}{g_{c}^{2}}\right)^{-2}$ respectively. Now we conclude with few observations. Firstly, the "non-abelian" hair of the black hole solution in the strong coupling limit possesses an exotic property. Unlike the abelian gauge charge in the familiar Einstein-Maxwell theory, the non-abelian gauge coupling parameter 
$g_{c}$ that characterizes the black hole solution above is not measurable as surface integrals at spatial infinity. This is because the metric solution is coupled to the vacuum gauge solution $F_{\mu \nu}=0$ in this EAT theory. Secondly, the metric solutions for cases (i) and (ii) are shown to exhibit the violation of cosmic censorship hypothesis. They, in fact, provide non-trivial counter-examples to the hypothesis in the sense that both the physical and the mathmatical versions of the hypothesis are violated. Namely, its classical metric solution turns out to violate the hypothesis while the EAT system itself satisfies the dominant energy condition (i.e., the locally non-negative matter energy density), $T_{\mu \nu} n^{\mu} n^{\nu}=1 / g_{c}^{2} r^{2} \geq 0$ (where $n^{\mu}$ is the timelike unit vector) on which the mathmatical version of the hypothesis is based. Note that the cosmic censorship hypothesis is believed to hold in the classical theory of general relativity. And thus far there has been no known concrete example of the violation of the hypothesis with its origin being at the classical theory. "White holes", whose existence has been proposed to be possible, should not be regarded as a counter -example to the "classical" cosmic censorship conjecture since they are objects that can be speculated to exist via the "time-reversal" of the classical black holes in the conventional definition or the quantum black holes that do evaporate in Hawking's option [7]. In this sense, our classical metric solution in EAT theory appears to be an interesting example that violates the hypothesis in the purely classical regime. It seems, however, fair to point out that the sort of the violation of the cosmic censorship hypothesis we found here is rather a peculiar consequence of the "exotic" classical metric solution that arises when a classical matter field theory is coupled to Einstein gravity than a phenomenologically realistic result arising from the gravitational collapse of some well-defined initial data.

\section{References}

[1] M.Kalb and P. Ramond, Phy. Rev. D9, 2273 (1974)

[2] E. Cremmer and J. Scherk, Nucl. Phys. B72, 117 (1974) 
[3] E. Sezgin and P. van Nieuwenhuizen, Phys. Rev. D22, 301 (1980)

[4] D. Z. Freedman and P. K. Townsend, Nucl. Phys. B177, 282 (1981)

[5] S. P. de Alwis et. al., Nucl. Phys. B303, 57 (1988); A. A. Slavnov and S. A. Frolov, Padova preprint DFPD 13/87.

[6] See, for instance, R. Rajaraman, Solitons and instantons (North-Holland Publishing company, Amsterdam, 1982)

[7] S. W. Hawking, Phys. Rev. D13, 191 (1976) 